\title{
Efecto de los rasgos de personalidad sobre el autocontrol en estudiantes universitarios de Argentina
}

\author{
Juan Ignacio Galli1 ${ }^{1}$ Macarena Verónica del Valle ${ }^{2}$, Eliana Vanesa Zamora ${ }^{3}$ y Lorena \\ Canet-Juric ${ }^{4}$
}

\section{RESUMEN}

Los rasgos de personalidad han sido propuestos como posibles factores que pueden contribuir a explicar las diferencias individuales en el autocontrol. Sin embargo, los resultados reportados en la literatura sobre el tema son, en cierta medida, contradictorios. El objetivo del presente estudio fue analizar el efecto de los cinco rasgos de personalidad sobre el autocontrol en estudiantes universitarios de Argentina. Los análisis de correlación evidenciaron un patrón de correlaciones positivas entre las puntuaciones en autocontrol y los rasgos responsabilidad y amabilidad, así como relaciones negativas entre neuroticismo y las medidas de autocontrol. A su vez, se presentaron correlaciones negativas entre el rasgo apertura a la experiencia y la dimensión control de los impulsos. Los análisis de regresión mostraron que los rasgos de personalidad en su conjunto explican el $43 \%$ de la varianza en la escala de autocontrol completa, el $28 \%$ de la varianza en la dimensión Control de los impulsos y el $42 \%$ de la varianza de la dimensión Autodisciplina, destacándose el rasgo responsabilidad como variable predictora de las puntuaciones en los tres casos.

Palabras clave: personalidad, autocontrol, estudiantes universitarios, evaluación.

\section{Effect of personality traits on self-control in university students from Argentina}

\begin{abstract}
Personality traits have been proposed as possible factors that may contribute to explain individual differences in self-control. However, the results reported in the literature on the subject are, to some extent, contradictory. The aim of the current study is to analyze the effect of personality traits on self-control in university students from Argentina. Correlation results demonstrated a pattern of positive correlations between self-control scores and conscientiousness and agreeableness traits, as well as negative correlations between neuroticism and self-control measures. In turn, there were negative correlations between openness to experience and the impulse control subscale. Regression analyzes showed that personality traits as a whole explain $43 \%$ of the variance on the full self-control scale, $28 \%$
\end{abstract}

\footnotetext{
${ }^{1}$ Instituto de Psicología Básica, Aplicada y Tecnología (IPSIBAT). Consejo Nacional de Investigaciones Cientificas y Técnicas (CONICET), Argentina; juani.galli8@gmail.com; http://orcid.org/ 0000-0002-87094913

2 Instituto de Psicología Básica, Aplicada y Tecnología (IPSIBAT). Consejo Nacional de Investigaciones Cientificas y Técnicas (CONICET), Argentina; eliana.zamora@conicet.gov.ar; http://orcid.org/0000-00026278-6665

3 Instituto de Psicología Básica, Aplicada y Tecnología (IPSIBAT). Consejo Nacional de Investigaciones Científicas y Técnicas (CONICET), Argentina; mdelvalle1989@gmail.com; https://orcid.org/0000-00033549-7224

4 Instituto de Psicología Básica, Aplicada y Tecnología (IPSIBAT). Consejo Nacional de Investigaciones Cientificas y Técnicas (CONICET), Argentina; lcanetjuric@gmail.com; https://orcid.org/0000-0003-41474889
} 
of the variance on the impulse control dimension, and $42 \%$ of the variance on the selfdiscipline dimension. The trait conscientiousness was, as expected, the most consistent predictor of scores on all three cases.

Keywords: personality, self-control, college students, assessment.

El autocontrol hace referencia a la capacidad que presentan las personas para regular sus pensamientos, emociones y conductas en presencia de un conflicto, entre la satisfacción de un deseo inmediato y un objetivo o meta de orden superior a largo plazo (Duckworth, Taxer, Eskreis-Winkler, Galla, \& Gross, 2019; Kotabe \& Hofmann, 2015). Así, el autocontrol puede conceptualizarse como un componente de los procesos autorregulatorios encargado de anular o modificar las respuestas prepotentes o automáticas, interrumpir tendencias comportamentales indeseadas y abstenerse de actuar en función de ellas (Hofmann \& Kotabe, 2012; Tangney, Baumeister, \& Boone, 2004). Se ha reportado que el autocontrol resulta fundamental para una adecuada adaptación a distintos ámbitos de la vida cotidiana (Galla \& Duckworth, 2015), entre los que se pueden destacar el educativo (Duckworth et al., 2019), el laboral (Wang, Chen, Dou, \& Liu, 2021) y el de las relaciones interpersonales (Unger, Bi, Xiao, \& Ybarra, 2016).

Se ha reportado que bajos niveles de autocontrol se asocian con una gran diversidad de problemáticas tanto a nivel individual como social, tales como el abuso de alcohol, nicotina y otras sustancias, la conducta antisocial, la procrastinación, las compras compulsivas y la mala alimentación (Friese \& Hofmann, 2009; Hay \& Meldrum, 2016; Herman \& Polivy, 2011; Mowlaie, Abolghasemi, \& Aghababaei, 2016). En cambio, niveles elevados de autocontrol se han asociado a beneficios en dominios como el rendimiento académico, las relaciones interpersonales, y el ajuste y el bienestar personal (Gillebaart \& Adriaanse, 2017; Tangney et al., 2004).

Especificamente, Tangney et al. (2004) dan cuenta de dos dimensiones fundamentales que contribuyen al autocontrol: la autodisciplina y el control de los impulsos. La autodisciplina se ha definido como la capacidad para resistir las tentaciones que pueden resultar inmediatamente gratificantes (i.e. dormir una siesta cuando deberíamos estudiar) y, en su lugar, persistir en actividades que no son inmediatamente gratificantes, pero son importantes para alcanzar metas u objetivos a largo plazo (i.e. aprobar un examen).

Por otro lado, el control de los impulsos refiere al nivel conductual en sentido estricto, y se define como la capacidad para cancelar o posponer actividades o experiencias positivas en el aquí y ahora. Mientras que la autodisciplina se encuentra más asociada al mantenimiento de hábitos, el control de los impulsos es un aspecto del autocontrol dedicado especialmente al control de las conductas impulsivas. En este sentido, del Valle, Galli, Urquijo y Canet Juric (2019) afirman que mientras el control de los impulsos se asocia con una restricción activa de la conducta sobre un estímulo saliente, la autodisciplina refiere a un constructo más general en el que, además, se incluye la capacidad para trabajar de forma eficiente y comprometida en la construcción de un plan y su seguimiento sostenido en el tiempo para alcanzar metas personales.

Ahora bien, las personas difieren en gran medida en su capacidad para ejercer el control sobre sus pensamientos, emociones y conductas. Para algunas 
personas, implementar el autocontrol parece demandar poco esfuerzo y suelen tener éxito. Sin embargo, para otras, el logro del autocontrol resulta más dificultoso, y los problemas para priorizar las metas a largo plazo anteponiéndose a los deseos o impulsos inmediatos se hacen evidentes (Hoyle \& Davisson, 2016).

Los rasgos de personalidad han sido propuestos como posibles factores que pueden contribuir a la existencia de diferencias individuales en el logro del autocontrol. Una de las teorías más difundidas sobre la personalidad es el Modelo de los Cinco Grandes Factores (Costa \& McCrae, 1992), que ha sido utilizado con frecuencia en las investigaciones que buscan analizar las relaciones entre la personalidad y el autocontrol. Dicho modelo plantea la existencia de cinco rasgos distinguibles entre sí: amabilidad (agreeableness), extraversión (extraversion), responsabilidad (conscientiousness), neuroticismo (neuroticism) y apertura a la experiencia (openness to experience). Estos rasgos se encuentran genéticamente determinados y, en la interacción con el medio y sus contingencias, condicionan la conducta individual (McCrae \& Costa, 2008). De manera resumida, el neuroticismo es la tendencia a experimentar emociones negativas; la amabilidad es la tendencia al altruismo y a la simpatía, y ayuda hacia otras personas; la responsabilidad refiere a la capacidad para ser organizado, riguroso y minucioso; la extraversión es la tendencia al gregarismo y al establecimiento de relaciones intensas y frecuentes con los demás; y, finalmente, la apertura a la experiencia es la preferencia por el mundo externo más que por el interno, por la búsqueda de nuevos desafios personales y la tendencia a visiones innovadoras (Sánchez \& Ledesma, 2007).

El estudio de las relaciones entre la personalidad y el autocontrol se ha centrado principalmente en las asociaciones entre el rasgo responsabilidad y el autocontrol. Específicamente, se ha sugerido que, tanto en términos conceptuales como empíricos, ambos constructos se encuentran estrechamente ligados y que existe cierto solapamiento entre los mismos (de Vries y van Gelder, 2013; Hoyle \& Davisson, 2016; Jones, 2017). Se ha señalado que las personas con altas puntuaciones en el rasgo responsabilidad se caracterizan por ser confiables, disciplinadas, ordenadas y organizadas (del mismo modo que son descriptas las personas con puntuaciones altas en autocontrol), mientras que las personas con puntuaciones bajas tienden a tener dificultades para controlar su comportamiento, presentan una mayor distractibilidad y son propensas a la procrastinación (Costa \& McCrae, 1992). En términos empíricos, distintos estudios han reportado correlaciones de moderadas a elevadas entre el rasgo responsabilidad y el autocontrol (e.g., $r=.66$, Bazzy, Woehr, \& Borns, 2017; $r=$ .55, De Vries \& Van Gelder, 2013; $r=.69$, Jones, 2017).

Sin bien resultan más escasos, otros estudios también han explorado las asociaciones entre el autocontrol y los restantes rasgos de personalidad (e.g., McCrae \& Löckenhoff, 2010; de Vries \& van Gelder, 2013), aunque los hallazgos resultan contradictorios en alguna medida. En este sentido, Olson (2005) ha sugerido que los rasgos de personalidad se pueden vincular conceptualmente con distintos dominios en los que puede ser necesario ejercer el autocontrol. Así, en el dominio interpersonal, el autocontrol estaría vinculado con la restricción de comportamientos hostiles y poco cooperativos (rasgo amabilidad); en el control orientado a la tarea, el autocontrol permitiría la restricción de comportamientos irresponsables y desadaptativos (rasgo responsabilidad); y en el dominio intrapersonal, el autocontrol permitiria la restricciones de las propias emociones negativas y estresantes (rasgo neuroticismo). En consonancia con los 
planteos de Olson (2005), Tangney et al. (2004) examinaron empíricamente las relaciones entre el autocontrol y los rasgos de personalidad mencionados y reportaron relaciones positivas significativas con responsabilidad $(r=.54)$, estabilidad emocional (el anverso de neuroticismo) $(r=.50) \mathrm{y}$, en menor medida, con amabilidad $(r=.29)$. No obstante, solo los rasgos responsabilidad y neuroticismo siguieron presentando relaciones significativas con el autocontrol cuando se controló la deseabilidad social. No se encontraron asociaciones entre el autocontrol y los rasgos extraversión y apertura a la experiencia. En el mismo sentido, Bazzy et al. (2017) reportaron correlaciones significativas directas entre el autocontrol y la responsabilidad $(r=.66)$ y la amabilidad $(r=.33)$, e inversas entre el primero y el neuroticismo $(r=-.42)$; la relación con responsabilidad fue la más marcada. Al igual que Tangney et al. (2004), no se hallaron correlaciones entre el autocontrol y los rasgos extraversión y apertura a la experiencia.

Sin embargo, otros autores han informado que los rasgos extraversión y apertura a la experiencia también presentan relaciones con el autocontrol. Por ejemplo, Pilarska (2018) no halló correlaciones entre autocontrol y extraversión, pero sí reportó asociaciones positivas entre autocontrol e intelecto (similar en contenido al rasgo apertura a la experiencia) $(r=.24)$. En contraposición, también se han informado relaciones negativas entre el autocontrol y el rasgo apertura a la experiencia $(r=-.27)$, así como entre el autocontrol y el rasgo extraversión ( $r=-.32$ ) (Green, O'Connor, Gartland, \& Roberts, 2015). Por su parte, Zhang et al. (2019) reportaron que el autocontrol presentó correlaciones negativas con neuroticismo $(r=-.57)$, y positivas con responsabilidad $(r=.74)$, amabilidad $(r=.68)$ y extraversión $(r=.48)$. A su vez, los autores indicaron que todos los rasgos resultaron predictores del autocontrol, a excepción de apertura a la experiencia. Sin embargo, Mao et al. (2018) informaron relaciones positivas, aunque bajas entre el autocontrol y la apertura a la experiencia $(r=.14)$, además de relaciones entre el primero y los restantes cuatro rasgos. Por lo tanto, las relaciones entre el autocontrol y los rasgos extraversión y apertura a la experiencia son aún poco claras tanto conceptual como empíricamente, destacándose la necesidad de realizar nuevos estudios a los fines de contribuir a una mejor compresión de las relaciones entre el autocontrol y dichos rasgos de personalidad.

En sintesis, los estudios previos que han abordado las relaciones entre los rasgos de personalidad y el autocontrol han reportado resultados dispares, marcando la necesidad de aportar nueva evidencia que contribuya a clarificar dichas relaciones. Si bien la mayor parte de los estudios destacan las correlaciones positivas elevadas entre el rasgo responsabilidad y el autocontrol, correlaciones positivas moderadas entre el rasgo amabilidad y autocontrol, y asociaciones negativas moderadas entre el rasgo neuroticismo y el autocontrol, existe el debate respecto a las asociaciones entre los restantes rasgos de personalidad y el autocontrol. Por tanto, el presente estudio tuvo como objetivo contribuir al estudio de las relaciones entre la personalidad y el autocontrol, analizando el efecto de los cinco rasgos de personalidad sobre el autocontrol en una muestra de estudiantes universitarios de Argentina. 


\section{MÉTODO}

\section{Participantes}

Se contó con la participación de 112 estudiantes de la Facultad de Psicología de la Universidad Nacional de Mar del Plata, con edades comprendidas entre los 18 y los 37 años $(M=23.05 ; D E=3.51)$. Del total, el $78.6 \%$ fueron mujeres $(n=88)$ y el $21.4 \%$ fueron hombres $(n=24)$. La distribución del género responde a las características poblacionales de la Facultad de Psicologia y de la Universidad en general, ya reportadas en otros estudios (e.g., del Valle et al., 2019).

\section{Materiales}

Autocontrol. Se administró la adaptación al español de la Escala de Autocontrol Abreviada (EAC-A) de Tangney et al. (2004; del Valle et al., 2019). El instrumento está conformado por 11 items que evalúan la capacidad de las personas para anular o modificar las respuestas prepotentes, y para interrumpir tendencias comportamentales indeseadas, absteniéndose de actuar en función de estas. Cuenta con dos factores diferenciados: uno de ellos, compuesto por 6 items, agrupa aspectos referidos a la capacidad de trabajar eficazmente y comprometerse con objetivos personales, denominado autodisciplina $(\mathrm{a}=.77)$; el otro factor, compuesto por 5 items, representa la capacidad general de la persona para refrenar conductas e impulsos y fue llamada control de los Impulsos $(a=.69)$. El instrumento se responde mediante una escala de tipo likert de cinco puntos, en donde el sujeto debe indicar desde 1 (nada) a 5 (mucho) en qué medida los ítems reflejan cómo es habitualmente. La adaptación argentina de del Valle et al. (2019) presentó un adecuado ajuste al modelo de dos factores $(C F I=.99 ; A G F I=.98 ; R M S E A=.035)$ e índices adecuados de confiabilidad (autodisciplina: $\mathrm{a}=.67$; Control de impulsos: $\mathrm{a}=$ .65). En este estudio, los indices de confiabilidad a fueron de .77 para autodisciplina, y .69 para control de impulsos. Además, el a para la escala total fue de .82 .

Personalidad. Se utilizó el Listado de Adjetivos para Evaluar la Personalidad (AEP; Ledesma, Sánchez, \& Diaz-Lázaro, 2011). El instrumento está compuesto por 67 items que expresan adjetivos descriptores de los rasgos del Modelo de los Cinco Grandes Factores de Personalidad (Big Five), como por ejemplo, melancólico, conservador, aventurero. Se indica al participante que califique cada adjetivo en función de cuánto lo describe, en una escala de tipo likert (desde 1 no me describe en absoluto a 5 me describe tal como soy). El instrumento posee la ventaja de haber sido desarrollado en el medio local y de requerir aproximadamente 10 minutos para ser contestado. Además, replica la estructura de cinco factores del Modelo de los Cinco Grandes, y ha mostrado buenas propiedades psicométricas en términos de validez y confiabilidad (amabilidad: a entre .75 y .84; neuroticismo: a entre .73 y .85; responsabilidad: a entre .73 y .80; extraversión: a entre .79 y .85; apertura a la experiencia: a entre .72 y .77; Ledesma et al., 2011; Sánchez \& Ledesma, 2007). En el presente estudio, los valores a fueron .71 para apertura a la experiencia, .85 para extraversión, .85 para responsabilidad, .80 para neuroticismo y .80 para amabilidad. 


\section{Procedimiento}

Se contactó a los estudiantes en actividades específicas vinculadas con la Universidad Nacional de Mar del Plata. Luego de explicarles las características del estudio, se los invitó a participar voluntariamente. Quienes accedieron debieron firmar un consentimiento informado. La toma de datos se realizó de manera grupal, y se llevó a cabo por los autores o fue supervisada por los mismos. Todos los procedimientos llevados a cabo respetaron y mantuvieron las recomendaciones de la American Psychological Association (2010), así como los principios éticos para la investigación con seres humanos de acuerdo con las reglamentaciones internacionales. Estos principios aseguran las necesarias condiciones de protección de la confidencialidad de los datos y del accionar en beneficio de los participantes del estudio.

\section{Análisis de los datos}

Los datos fueron sistematizados en una única base de datos y analizados mediante el programa SPSS (v. 23). No se registraron datos perdidos en el presente estudio. Se realizaron análisis estadísticos descriptivos (mínimo, máximo, curtosis, asimetría, media y desvío estándar) para caracterizar las variables bajo estudio. Se comprobó la normalidad de la distribución de las variables mediante la prueba Shapiro-Wilk y no resultó significativo el valor para ninguna de las variables bajo estudio $(p>.01)$. También, se verificó la normalidad de las variables considerando el criterio que establece que valores de asimetria y curtosis comprendidos entre \pm 2 puntos se consideran limites aceptables de dicha distribución (e.g., George \& Mallery, 2016; Trochim \& Donnelly, 2006). Se exploró la existencia de casos atípicos siguiendo el criterio de Tabachnick y Fidell (2001) de 3.29 desvíos más allá de la media y se detectó la presencia de un caso atípico univariado (control de impulsos: -3.38). No obstante, este fue conservado puesto que su eliminación no afectaba la normalidad de las variables ni los resultados.

Para determinar la asociación entre los rasgos de personalidad y el autocontrol, se aplicaron correlaciones $r$ de Pearson. Para estimar el tamaño del efecto de las correlaciones, se siguió el criterio de Cohen (1988) que indica que las correlaciones de .10, .30 y .50 pueden considerarse respectivamente como pequeñas, moderadas y grandes. Considerando los valores de confiabilidad de algunas de las dimensiones analizadas (e.g., Control de impulsos: $a=.69$ ), se calculó la corrección por atenuación de los coeficientes de las correlaciones entre personalidad y autocontrol, y se estimó el porcentaje de atenuación en cada caso (Domínguez-Lara, 2017). También, se analizó si existían diferencias en función del género de los participantes mediante la aplicación de pruebas $t$ de Student. Para determinar si existían asociaciones entre la edad de los participantes y las variables bajo estudio, se aplicaron correlaciones $r$ de Pearson. Luego, para determinar el efecto de cada rasgo de personalidad sobre el autocontrol, se analizaron tres modelos de regresión lineal múltiple, mediante el método entrar (enter). En todos los casos, las variables independientes introducidas fueron los cinco rasgos de personalidad. En el primer modelo, la variable dependiente fue el puntaje total en la escala de autocontrol; en el segundo modelo, la variable dependiente fue la dimensión control de los Impulsos; y en el tercer modelo, la variable dependiente fue la dimensión autodisciplina. Los diagnósticos de colinealidad de los modelos de regresión mostraron valores (VIF) inferiores a 1.28 para todas las variables independientes. La distribución de los residuos de los tres modelos de regresión 
también resultó normal (Shapiro-Wilk $=p>.01$ ). El tamaño del efecto de los modelos se estimó mediante $f^{2}$ y considerando $.10, .25$ y .45 como efectos pequeños, medianos y grandes, respectivamente (Cohen, 1988). Asimismo, se calculó la potencia de los modelos mediante el programa G*Power (Cárdenas Castro \& Arancibia Martini, 2014; Faul, Erdfelder, Buchner, \& Lang, 2009).

\section{RESULTADOS}

En la Tabla 1, se presentan los estadísticos descriptivos para los rasgos de personalidad y la escala de autocontrol completa, y sus subescalas (autodisciplina y control de los impulsos). En esa misma tabla, también se presentan los índices de correlación entre las variables estudiadas. Teniendo en cuenta los valores de confiabilidad de algunas de las dimensiones analizadas (e.g., control de los impulsos: a = .69), se calcularon los coeficientes de las correlaciones entre personalidad y autocontrol considerando la corrección por atenuación. Los porcentajes de atenuación se presentan en la Tabla 2.

Tabla 1.

Estadísticos descriptivos y correlaciones entre las variables estudiadas.

\begin{tabular}{lcccccccc}
\hline & 1 & 2 & 3 & 4 & 5 & 6 & 7 & 8 \\
\hline 1. Extraversión & & & & & & & & \\
2. Amabilidad & $.27^{* *}$ & & & & & & & \\
3. Responsabilidad & $.21^{*}$ & $.29^{* *}$ & & & & & & \\
4. Neuroticismo & $-.17^{*}$ & $-.23^{* *}$ & $-.40^{* *}$ & & & & & \\
5. Apertura a la experiencia & $.25^{* *}$ & .12 & .08 & .10 & & & & \\
6. Autodisciplina & .13 & $.20^{*}$ & $.62^{* *}$ & $-.41^{* *}$ & -.06 & & & \\
7. Control de los Impulsos & .09 & $.29^{* *}$ & $.43^{* *}$ & $-.22^{* *}$ & $-.21^{*}$ & $.57^{* *}$ & & \\
8. Autocontrol total & .12 & $.28^{* *}$ & $.59^{* *}$ & $-.36^{* *}$ & -.16 & $.89^{* *}$ & $.88^{* *}$ & \\
\hline ME & 3.59 & 3.78 & 3.56 & 2.95 & 3.38 & 3.28 & 3.58 & 3.43 \\
DE & 0.67 & 0.44 & 0.56 & 0.57 & 0.54 & 0.71 & 0.70 & 0.62 \\
Mínimo & 1.80 & 2.56 & 2.38 & 1.22 & 1.90 & 1.50 & 1.20 & 1.35 \\
Máximo & 5.00 & 4.88 & 5.00 & 4.33 & 4.80 & 5.00 & 5.00 & 4.73 \\
Asimetría & -0.18 & -0.15 & 0.00 & -0.11 & 0.14 & 0.02 & -0.36 & -0.32 \\
Curtosis & -0.45 & 0.80 & -0.54 & -0.08 & 0.19 & -0.41 & 0.41 & 0.28 \\
\hline
\end{tabular}

Nota: ${ }^{*} p<.05 ;{ }^{* *} p<.01$

Tabla 2.

Porcentajes de correcciones por atenuación.

\begin{tabular}{lccc}
\hline & $\begin{array}{c}\boldsymbol{r} \\
\text { observada }\end{array}$ & $\begin{array}{c}\boldsymbol{r} \\
\text { verdadera }\end{array}$ & $\begin{array}{c}\% \\
\text { atenuación }\end{array}$ \\
\cline { 2 - 4 } Extraversión - Autodisciplina & .13 & .16 & $19 \%$ \\
Extraversión - Control de Impulsos & .09 & .12 & $25 \%$ \\
Extraversión - AC total & .12 & .14 & $14 \%$ \\
Amabilidad - Autodisciplina & .20 & .25 & $20 \%$ \\
Amabilidad - Control de Impulsos & .29 & .39 & $26 \%$ \\
Amabilidad - AC total & .28 & .35 & $20 \%$ \\
Responsabilidad - Autodisciplina & .62 & .77 & $19 \%$ \\
Responsabilidad - Control de Impulsos & .43 & .56 & $23 \%$ \\
Responsabilidad - AC total & .59 & .71 & $17 \%$ \\
Neuroticismo - Autodisciplina & -.41 & -.52 & $21 \%$ \\
Neuroticismo - Control de Impulsos & -.22 & -.30 & $27 \%$ \\
Neuroticismo - AC total & -.36 & -.44 & $18 \%$ \\
Apertura a la experiencia - Autodisciplina & -.06 & -.08 & $25 \%$ \\
Apertura a la experiencia - Control de Impulsos & -.21 & -.30 & $30 \%$ \\
Apertura a la experiencia - Autocontrol Escala Total & -.16 & .21 & $24 \%$ \\
\hline
\end{tabular}


Se observaron correlaciones positivas entre el rasgo responsabilidad y los indicadores de autocontrol. Estas resultaron entre moderadas y altas. El rasgo neuroticismo mostró asociaciones inversas entre bajas y moderadas con los niveles de autocontrol. El rasgo amabilidad también mostró asociaciones entre bajas y moderadas, aunque las mismas fueron directas. El rasgo apertura mostró una correlación negativa baja con la subescala control de los impulsos. No se encontraron correlaciones entre el rasgo extraversión y las medidas de autocontrol. Los porcentajes de atenuación sugieren que las correlaciones se encuentran parcialmente afectadas por los errores de medida de los instrumentos, siendo en la realidad mayores a lo observado.

A continuación, se realizaron análisis de regresión lineal múltiple para determinar qué factores de personalidad predecian significativamente las puntuaciones en autocontrol. Dado que no se observaron diferencias significativas en función del género para ninguna de las variables bajo estudio y que tampoco se hallaron correlaciones asociadas a la edad de los participantes, dichas variables no fueron incluidas en los modelos de regresión. Los resultados se presentan en la Tabla 3. Los tres modelos resultaron estadísticamente significativos y presentaron un tamaño del efecto grande. El valor de la potencia estadística fue alto para los tres modelos, limitando la posibilidad de cometer errores de tipo II. Los factores de personalidad en su conjunto explican el 43\% de la varianza en la escala de autocontrol completa ( $r^{2}$ $=.42 ; r^{2}$ corregida $\left.=.39 ; F_{(5,106)}=16.16 ; p<.001\right)$, el $28 \%$ de la varianza en la dimensión control de los impulsos $\left(r^{2}=.28 ; r^{2}\right.$ corregida $=.25 ; F_{(5,106)}=8.42 ; p$ $<.001)$, y el $42 \%$ de la varianza de la dimensión autodisciplina $\left(r^{2}=.42 ; r^{2}\right.$ corregida $\left.=.40 ; F_{(5,106)}=15.52 ; p<.001\right)$. Se destaca la importancia del rasgo responsabilidad como variable predictora de las puntuaciones tanto de la escala de autocontrol completa como de las dimensiones control de los impulsos y autodisciplina. En menor medida, apertura a la experiencia y amabilidad aportaron a la varianza del control de los impulsos, mientras que neuroticismo resultó un predictor para la autodisciplina. Las correlaciones respaldan lo reportado por los coeficientes estandarizados acerca de la varianza única explicada por cada una de estas variables independientes.

Tabla 3.

Análisis de regresión múltiple de los rasgos de personalidad sobre los niveles de autocontrol.

\begin{tabular}{|c|c|c|c|c|c|c|c|c|c|}
\hline & \multicolumn{3}{|c|}{ AC-breve Total } & \multicolumn{3}{|c|}{ Control de impulsos } & \multicolumn{3}{|c|}{ Autodisciplina } \\
\hline $\begin{array}{r}r^{2} \\
F_{(105)} \\
f^{2} \\
1-\beta\end{array}$ & & $\begin{array}{c}.43 \\
16.16^{\star *} \\
0.76 \\
1.00\end{array}$ & & & $\begin{array}{c}.28 \\
8.42^{* \star} \\
0.40 \\
0.99\end{array}$ & & & $\begin{array}{c}.42 \\
15.52^{* *} \\
0.73 \\
1.00\end{array}$ & \\
\hline \multirow{2}{*}{$1-\beta$} & \multirow[b]{2}{*}{$\beta$} & \multicolumn{2}{|c|}{ Correlaciones } & \multirow[b]{2}{*}{$\beta$} & \multicolumn{2}{|c|}{ Correlaciones } & \multirow[b]{2}{*}{$\beta$} & \multicolumn{2}{|c|}{ Correlaciones } \\
\hline & & Parc. & $\begin{array}{l}\text { Semi- } \\
\text { parc. }\end{array}$ & & Parc. & $\begin{array}{l}\text { Semi- } \\
\text { parc. }\end{array}$ & & Parc. & $\begin{array}{l}\text { Semi- } \\
\text { parc. }\end{array}$ \\
\hline Extraversión & .04 & .05 & .03 & .02 & .02 & .02 & -.01 & -.01 & -.01 \\
\hline Amabilidad & .14 & .17 & .13 & $.20^{*}$ & .22 & .19 & .01 & .01 & .01 \\
\hline Responsabilidad & $.54^{* *}$ & .53 & .47 & $.39^{* *}$ & .38 & .35 & $.55^{* *}$ & .54 & .49 \\
\hline Neuroticismo & -.10 & -.11 & -.09 & .01 & .01 & .01 & $-.18^{*}$ & -.20 & -.16 \\
\hline Apertura a la experiencia & $-.19^{*}$ & -.23 & -.18 & $-.28^{* *}$ & -.30 & -.26 & -.09 & -.11 & -.09 \\
\hline
\end{tabular}

Nota: ${ }^{*} p<.05 ;{ }^{* *} p<.01$; se presentan los $\beta$ estandarizados; Parc. = Correlaciones parciales; Semi-parc = Correlaciones semi-parciales. 


\section{DISCUSIÓN}

Se ha sugerido que las personas difieren en su capacidad para controlar sus impulsos y que, en parte, tales diferencias pueden estar asociadas con distintas facetas o rasgos de personalidad (de Vries \& van Gelder, 2013). De esta manera, este trabajo se propuso analizar la capacidad predictiva de ciertos rasgos de personalidad sobre la capacidad de autocontrol.

Los resultados permitieron mostrar que los rasgos con mayor efecto sobre el autocontrol fueron responsabilidad, apertura a la experiencia, amabilidad y neuroticismo. El efecto del primero de ellos fue mucho más importante que el de los restantes. El papel del rasgo responsabilidad como predictor del autocontrol hallado en este estudio se encuentra en línea con estudios previos (e.g., de Vries \& van Gelder, 2013; Pilarska, 2018). Este rasgo resultó un mejor predictor del factor autodisciplina que de control de los impulsos, lo que resulta esperable dado que se vincula con la capacidad para trabajar disciplinadamente y con esfuerzo en la concreción de metas y objetivos valiosos (Ledesma et al., 2011). Así, la responsabilidad explica la capacidad de las personas para persistir en tareas o actividades que no son inmediatamente gratificantes, pero que sí son importantes para alcanzar metas u objetivos a largo plazo. No obstante, cabe señalar que es posible que las asociaciones entre el rasgo responsabilidad y la autodisciplina puedan deberse a las similitudes conceptuales entre ambos constructos, lo que se manifiesta en un alto grado de superposición en el contenido de los ítems que se incluyen en los instrumentos (Hoyle \& Davisson, 2016).

Además, el rasgo amabilidad, que se vincula con tendencias altruistas y prosociales, resultó predictor de la dimensión control de los impulsos. La asociación entre dichas variables puede deberse a que, a los fines de establecer vínculos interpersonales duraderos, es necesario inhibir ciertas tendencias automáticas o conductas impulsivas que puedan repercutir negativamente en el vínculo con otras personas (Hoyle \& Davisson, 2016). De hecho, las conductas ligadas al no control de los impulsos (i.e., decir cosas inapropiadas o responder a estímulos salientes divertidos o gratificantes), tienen referencia directa con los comportamientos implicados en la interacción con otras personas.

El rasgo neuroticismo resultó predictor solo de la autodisciplina, pero no del control de los impulsos. En este sentido, las personas que poseen mayor inestabilidad emocional y una mayor tendencia a experimentar emociones negativas presentan menor capacidad para concentrarse y persistir en tareas o actividades que no son inmediatamente gratificantes, pero que son importantes para alcanzar metas $\mathrm{u}$ objetivos a largo plazo. Al respecto, diversos estudios (e.g., Cervera et al., 2003; Kozak \& Fought, 2011; Terracciano, Löckenhoff, Crum, Bienvenu, \& Costa, 2008; Wilson, Sayette, \& Fiez, 2014) sugieren que la experimentación de emociones negativas y la incapacidad de tolerarlas, promueven conductas adictivas y desadaptativas, como fumar, comer en exceso o consumir drogas.

En consonancia con algunos estudios previos (e.g., Bazzy et al., 2017; de Vries \& van Gelder, 2013; Pilarska, 2018: Tangney et al., 2004) y en contraste con otros (e.g., Mao et al., 2018; Zhang et al., 2019), no se encontraron asociaciones entre el rasgo extraversión y la escala de autocontrol y sus factores. En general, se ha sugerido que aquellas personas con mayores niveles de extraversión presentan una tendencia al gregarismo y a la búsqueda de sensaciones. Sin embargo, los resultados del presente estudio se suman a 
aquellos que no han encontrado asociaciones entre el rasgo extraversión y la capacidad para controlar las emociones, pensamientos y conductas.

Por último, en disonancia con algunos estudios previos (e.g., Bazzy et al., 2017; de Vries \& van Gelder, 2013; Tangney et al., 2004) el rasgo apertura a la experiencia resultó predictor de las puntuaciones en la escala de autocontrol y, especialmente, en el factor control de los impulsos. En línea con los resultados obtenidos, se ha sugerido que mayores niveles de apertura a la experiencia, vinculada con la tendencia a realizar actividades novedosas y experimentar nuevas sensaciones, podrian estar asociados a una menor capacidad para el control de los impulsos (De Young \& Rueter, 2016). Específicamente, se han reportado asociaciones entre el rasgo apertura a la experiencia y el constructo falta de premeditación (Kaufman, 2013), definido como la tendencia a actuar sin reflexionar previamente sobre las posibles consecuencias de las acciones (Whiteside \& Lynam, 2001).

Si bien se han informado diferencias en los rasgos de personalidad en función del género (Schmitt et al., 2017), no se registraron diferencias significativas asociadas al género de los participantes para ninguna de las variables analizadas en el estudio. Por tal motivo, el género no fue incluido en los modelos de regresión. La ausencia de diferencias en función del género se ha reportado en estudios recientes con poblaciones similares (Khalil, del Valle, Zamora, \& Urquijo, 2020), y puede estar vinculada con características propias de los estudiantes universitarios. A su vez, dado que la muestra es homogénea en el sentido de que se trata exclusivamente de estudiantes de una única unidad académica, es posible que la elección de una carrera universitaria esté influenciada, al menos en parte, por ciertos rasgos de personalidad (Benítez \& Pérez, 2011).

En suma, este estudio representa un aporte al debate presente en la literatura respecto a las asociaciones entre los rasgos de personalidad y el autocontrol. Supone, además, un insumo teórico a la comprensión del fenómeno del autocontrol, sobre todo teniendo en cuenta la importancia y el poder predictivo del autocontrol sobre la adaptación del individuo al ambiente (Galla \& Duckworth, 2015). Sin embargo, la presente investigación presenta limitaciones. En primer lugar, la muestra es pequeña y está formada exclusivamente por estudiantes, mayoritariamente mujeres, de una unidad académica en particular, lo que limita la generalización de los resultados. En segundo lugar, otra limitación está vinculada con la selección de los instrumentos, ya que solo se incluyeron medidas de cuestionario para evaluar tanto rasgos de personalidad como de autocontrol. Estudios futuros deberían incluir una muestra más grande y heterogénea (mayor número de estudiantes universitarios de distintas unidades académicas). A su vez, sería recomendable incluir medidas adicionales de autocontrol, tanto cuestionarios como medidas comportamentales, dado que existen diferentes modalidades de evaluación de dicho constructo que podrian resultar complementarias y enriquecer, así, el análisis de las relaciones entre el autocontrol y los rasgos de personalidad.

En síntesis, más allá de las limitaciones del estudio, los hallazgos son en su mayoría consistentes con las propuestas teóricas y los resultados empíricos de otros estudios. Asimismo, contribuyen al análisis de la incidencia de los cinco rasgos de personalidad sobre la conducta humana; específicamente, sobre la habilidad para regular los pensamientos, emociones y conductas en momentos en los que la satisfacción de un deseo inmediato se encuentra en conflicto con un objetivo o meta a largo plazo (Duckworth et al., 2019). 


\section{REFERENCIAS}

American Psychological Association. (2010). Ethical principles of psychologists and code of conduct. Washington, DC: The American Psychologist. Retrieved from www.apa.org/ethics/code/principles.pdf

Bazzy, J. D., Woehr, D. J., \& Borns, J. (2017). An Examination of the Role of Self-Control and Impact of Ego Depletion on Integrity Testing. Basic and Applied Social Psychology, 39(2), 101-111. https://doi.org/10.1080/01973533.2017.1283502

Benítez, J. T. B., \& Pérez, D. L. C. (2011). Personalidad y toma de decisiones vocacionales en universitarios. Relieve: Revista Electrónica de Investigación y Evaluación Educativa, 17(1), 1-15.

Cárdenas Castro, M. \& Arancibia Martini, H. (2014). Potencia estadística y cálculo del tamaño del efecto en G*Power: complementos a las pruebas de significación estadística y su aplicación en psicología. Salud $\&$ Sociedad, 5(2), 210-244. https://doi.org/10.22199/S07187475.2014.0002.00006

Cervera, S., Lahortiga, F., Angel Martínez-González, M., Gual, P., Irala-Estévez, J. D., \& Alonso, Y. (2003). Neuroticism and low self-esteem as risk factors for incident eating disorders in a prospective cohort study. International Journal of Eating Disorders, 33(3), 271-280. https://doi.org/10.1002/eat.10147

Cohen, J. (1988). Statistical power analysis for the behavioral sciences (2nd ed.). Hillsdale, NJ: Erlbaum.

Costa, P. T. \& McCrae, R. R. (1992). Four ways five factors are basic. Personality and Individual Differences, 13, 653-665. https://doi.org/10.1016/0191-8869(92)90236-I

del Valle, M., Galli, J. I., Urquijo, S., \& Canet Juric, L. (2019). Adaptación al español de la Escala de Autocontrol y de la Escala de Autocontrol-Abreviada y evidencias de validez en población universitaria. Revista Argentina de Ciencias del Comportamiento, 11(2), 52-64. https://doi.org/10.32348/1852.4206.v11.n2.23413

de Vries, R. E. \& van Gelder, J. L. (2013). Tales of two self-control scales: Relations with Five-Factor and HEXACO traits. Personality and Individual Differences, 54(6), 756-760. https://doi.org/10.1016/j.paid.2012.12.023

De Young, C. G. \& Rueter, A. R. (2016). Impulsivity as a personality trait. En K. Vohs \& R. Baumeister (Eds.) Handbook of Self-Regulation. Research, Theory, and Applications, 3rd Edition (pp. 345-363). New York: The Guilford Press.

Dominguez-Lara, S. (2017). Atenuación de correlaciones y baja fiabilidad: ¿realmente importa?. Nutrición Hospitalaria, 34(5), 1261-1262. https://doi.org/10.20960/nh.1391

Duckworth, A. L., Taxer, J. L., Eskreis-Winkler, L., Galla, B. M., \& Gross, J. J. (2019). Self-Control and Academic Achievement. Annual Review of Psychology, 70, 373-399. https://doi.org/10.1146/annurevpsych-010418-103230

Faul, F., Erdfelder, E., Buchner, A., \& Lang, A. G. (2009). Statistical power analyses using G*Power 3.1: Tests for correlation and regression analyses. Behavior Research Methods, 41, 1149-1160. https://doi.org/10.3758/BRM.41.4.1149

Friese, M. \& Hofmann, W. (2009). Control me or I will control you: Impulses, trait self-control, and the guidance of behavior. Journal of Research in Personality, 43, 795-805. https://doi.org/10.1016/j.jrp.2009.07.004

Galla, B. M. \& Duckworth, A. L. (2015). More than resisting temptation: Beneficial habits mediate the relationship between self-control and positive life outcomes. Journal of Personality and Social Psychology, 109(3), 508-525. https://doi.org/10.1037/pspp0000026

Gillebaart, M. \& Adriaanse, M. A. (2017). Self-control predicts exercise behavior by force of habit, a conceptual replication of Adriaanse et al. (2014). Frontiers in Psychology, 8(FEB), 1-6. https://doi.org/10.3389/fpsyg.2017.00190

George, D. \& Mallery, P. (2016). IBM SPSS statistics 23 step by step: A simple guide and reference (14th Ed.). UK: Routledge. https://doi.org/10.4324/9781315545899

Green, J. A., O'Connor, D. B., Gartland, N., \& Roberts, B. W. (2016). The Chernyshenko Conscientiousness Scales: A New Facet Measure of Conscientiousness. Assessment, 23(3), 374-385. https://doi.org/10.1177/1073191115580639

Hay, C. \& Meldrum, R. (2016). Self-control and crime over the life course. Los Angeles: Sage. https://doi.org/10.4135/9781483397726

Herman, C. P. \& Polivy, J. (2011). Self-regulation and the obesity epidemic. Social Issues and Policy Review, 5(1), 37-69. https://doi.org/10.1111/j.1751-2409.2011.01025.x

Hofmann, W. \& Kotabe, H. (2012). A General Model of Preventive and Interventive Self-Control. Social and Personality Psychology Compass, 10, 707-722. https://doi.org/10.1111/j.1751-9004.2012.00461.x

Hoyle, R. H. \& Davisson, E. K. (2016). Varieties of self-control and their personality correlates. En K. Vohs \& R. Baumeister (Eds.) Handbook of Self-Regulation. Research, Theory, and Applications, 3rd Edition (pp. 396-413). New York: The Guilford Press.

Jones, S. (2017). Does choice of measure matter? Assessing the similarities and differences among self-control scales. Journal of Criminal Justice, 50, 78-85. https://doi.org/10.1016/j.jcrimjus.2017.04.005

Kaufman, S. B. (2013). Opening up openness: A four- factor model of openness to experience and creative achievement in the arts and sciences. Journal of Creative Behavior, 47, 233-255. https://doi.org/10.1002/jocb.33

Khalil, Y., del Valle, M. V., Zamora, E. V., \& Urquijo, S. (2020). Dificultades de regulación emocional y bienestar psicológico en estudiantes universitarios. Subjetividad y Procesos Cognitivos, 24(1), 69-83. https://doi.org/10.18682/pd.v20i1.1877

Kotabe, H. P. \& Hofmann, W. (2015). On Integrating the Components of Self-Control. Perspectives on Psychological Science, 10(5), 618-638. https://doi.org/10.1177/1745691615593382

Kozak, A. T. \& Fought, A. (2011). Beyond alcohol and drug addiction. Does the negative trait of low distress tolerance have an association with overeating?. Appetite, 57(3), 578-581. 
https://doi.org/10.1016/j.appet.2011.07.008

Ledesma, R. D., Sánchez, R., \& Díaz-Lázaro, C. M. (2011). Adjective checklist to assess the big five personality factors in the Argentine population. Journal of Personality Assessment, 93(1), 46-55. https://doi.org/10.1080/00223891.2010.513708

Mao, T., Pan, W., Zhu, Y., Yang, J., Dong, Q., \& Zhou, G. (2018). Self-control mediates the relationship between personality trait and impulsivity. Personality and Individual Differences, 129(378), 70-75. https://doi.org/10.1016/j.paid.2018.03.013

McCrae, R. R. \& Costa, P. T., Jr. (2008). The five-factor theory of personality. En O. P. John, R. W. Robins, \& L. A. Pervin (Eds.), Handbook of personality: Theory and research (pp. 159-181). (3rd ed.). New York: Guilford Press.

McCrae, R. R. \& Löckenhoff, C. E. (2010). Self-Regulation and the Five-Factor Model of Personality Traits. En R. Hoyle (Ed.), Handbook of Personality and Self-Regulation (pp.145-168). Hoboken: Wiley Blackwell. https://doi.org/10.1002/9781444318111.ch7

Mowlaie, M., Abolghasemi, A., \& Aghababaei, N. (2016). Pathological narcissism, brain behavioral systems and tendency to substance abuse: The mediating role of self-control. Personality and Individual Differences, 88, 247-250. https://doi.org/10.1016/j.paid.2015.09.019

Olson, K. R. (2005). Engagement and Self-Control: Superordinate dimensions of Big Five traits. Personality and Individual Differences, 38(7), 1689-1700. https://doi.org/10.1016/j.paid.2004.11.003

Pilarska, A. (2018). Big-Five personality and aspects of the self-concept: Variable- and person-centered approaches. Personality and Individual Differences, 127(July 2017), 107-113. https://doi.org/10.1016/j.paid.2018.01.049

Sánchez, R. \& Ledesma, R. (2007). Los Cinco Grandes Factores: cómo entender la personalidad y cómo evaluarla. En A. Monjeau (Ed.), Conocimiento para la transformación. Serie Investigación y Desarrollo (pp. 131-160). Buenos Aires: Ediciones Universidad Atlántida Argentina.

Schmitt, D. P., Long, A. E., McPhearson, A., O’Brien, K., Remmert, B., \& Shah, S. H. (2017). Personality and gender differences in global perspective. International Journal of Psychology, 52, 45-56. https://doi.org/10.1002/ijop.12265

Tangney, J. P., Boone, A. L., \& Baumeister, R. F. (2004). High self-control predicts good adjustment, less pathology, better grades, and interpersonal success. Journal of Personality, 72(3), 271-324. https://doi.org/10.1111/j.0022-3506.2004.00263.x

Tabachnick, B. G. \& Fidell, L. S. (2001). Using Multivariate Statistics (4th ed.). Boston: Allyn \& Bacon

Terracciano, A., Löckenhoff, C. E., Crum, R. M., Bienvenu, O. J., \& Costa, P. T. (2008). Five-Factor Model personality profiles of drug users. BMC Psychiatry, 8(1), 22. https://doi.org/10.1186/1471-244X-8-22

Trochim, W. M. \& Donnelly, J. P. (2006). The research methods knowledge base (3rd ed.). Atomic Dog.

Unger, A., Bi, C., Xiao, Y. Y., \& Ybarra, O. (2016). The revising of the Tangney Self-Control Scale for Chinese students. PsyCh Journal, 5(2), 101-116. https://doi.org/10.1002/pchj.128

Wang, Y. J., Chen, K. Y., Dou, K., \& Liu, Y. Z. (2021). Linking Self-Control to Voluntary Behaviors at Workplace: The Mediating Role of Job Satisfaction. Frontiers in Psychology, 12(March), 1-9. https://doi.org/10.3389/fpsyg.2021.530297

Whiteside, S. P. \& Lynam, D. R. (2001). The Five Factor Model and impulsivity: Using a structural model of personality to understand impulsivity. Personality and Individual Differences, 30(4), 669-689. https://doi.org/10.1016/S0191-8869(00)00064-7

Wilson, S. J., Sayette, M. A., \& Fiez, J. A. (2014). Self-control, negative affect and neural activity during effortful cognition in deprived smokers. Social Cognitive and Affective Neuroscience, 9(6), 887-894. https://doi.org/10.1093/scan/nst065

Zhang, G., Chen, X., Xiao, L., Li, Y., Li, B., Yan, Z., ... Rost, D. H. (2019). The relationship between big five and self-control in boxers: A mediating model. Frontiers in Psychology, 10, 1690. https://doi.org/10.3389/fpsyg.2019.01690

Recibido 25-02-2021 | Aceptado 27-05-2021 